\title{
UV Transient Luminous Events observed with a terrestrial pinhole
}

\section{S. Hernandez, E. Ponce ${ }^{* \dagger}$}

Autonomous University of Puebla, Mexico. 4 sur 104 Col. Centro C.P. 72000, Puebla, Mexico.

E-mail: shernandez@fcfm.buap.mx, eponcedfcfm.buap.mx

The current generation of experiments devoted to study extreme energy cosmic rays will be at space platforms. Recent satellite missions have shown that the UV light background is more complex than previous models. Therefore, the observation of transient luminous events TLEs at the upper atmosphere will be important. Information about the time and space evolution of this very fast events may need to be recorded, this impose requirements of a wide field of view and the largest focus depth. The simplest optical design, a pinhole camera fulfills this characteristics. This pinhole camera have a multianode photomultiplier $8 \times 8$ pixels, that allow us to register $2 \mathrm{~d}$ images of TLEs of about milliseconds. In this work we present the shape of some events recorded from Sierra Negra Volcano in Mexico and its capabilities in order to use it as a monitoring device.

36th International Cosmic Ray Conference -ICRC2019-

July 24th - August 1st, 2019

Madison, WI, U.S.A.

* Speaker.

${ }^{\dagger}$ A footnote may follow. 


\section{Introduction}

One of the important phenomena in night atmosphere, related to ultraviolet light background measurement, are TLE (transient luminous events) characterized by very bright (energy in UV up to 0.1-1 MJ) short (duration of 1-300 ms) flashes. The first global measurements of UV flashes were done by two artificial microsatellites designed for scientific and educational purposes Universitetskii-Tat'yana and Universitetskii-Tat'yana 2; recently, continue with the observations of UV radiation (300-400 nm wavelength) bursts in the night atmosphere of the Earth with TUS experiment on-board Lomonosov satellite. These scientific Earth satellites were designed and constructed at the MSU Skobeltsyn Institute of Nuclear Physics (SINP) (Moscow). In this project we present a method of TLE measurement by the imaging pinhole camera. The presented pinhole camera is planned for operation in space experiments devoted to study of processes of electron acceleration in the atmosphere, electric discharges and relativistic electron precipitation to the atmosphere from the magnetosphere among others.

\section{Pinhole camera}

The Camera obscura detector is the one of best optical imaging designs due to their simplicity wide field of view and large deep field [1]. We plan to operate it onboard a space mission satellite planed to orbit the earth at $700 \mathrm{Km}$ height. The camera obscura it will be used to explore the eventual creation of perturbations near to the event that may should trigger some TLE's. This perturbation was considerated as background noise in the tracking of the cosmic ray original particle. The high brightness of TLE may allow us to use the simplest pinhole optics for measuring the image in pixels of UV detector. The optimal imaging quality in a pinhole camera is achieved if the hole size is equal to the detector pixel size. Our aim is to measure not only the TLE image but also the temporal profile of the image with time resolution of about milliseconds. Today such a fast photo detector is available only as a Multi-Anode Photomultiplier Tube (MaPMT) with some number of pixels. The size of the pixel in MaPMT is of about 2-3 mm. Assuming the camera hole equal to this size [2] and taking the TLE UV intensity and time duration from [3], it is possible to estimate the signals in the pixels of the pinhole camera. Efficiency of the MaPMT pixels to UV is around $20 \%$ for wavelengths $\lambda=300-400 \mathrm{~nm}$ and decreases below $\lambda=300 \mathrm{~nm}$. For estimate of the TLE image signals the lateral distribution of UV intensity during the event is needed. We assume that UV flash images cover a circular area of some tens of $\mathrm{km}$ diameter (as it was measure in a typical TLE by video cameras, figure 1) with uniform intensity over the circle. So the total UV energy Euv radiated by the TLE correspond to the number of photons of wavelength $\lambda=300-400$ $\mathrm{nm}$. To improve the detection of TLEs, for this project we considered the configuration where the TLE whole area is observed by all pixels, in this setup a detailed image of the UV flash in space and time is obtained as it was suggested in [1] for EAS Cherenkov light observations. A scheme of the designed camera obscura it is shown in figure 2. In our case the design and construction of the camera obscura was made with the following technical parameters: one photo receivers MaPMT model H7546B (Hamamatsu) a matrix of 8 by 8 pixels with a pixel size of $2 \times 2 \mathrm{~mm}$. The pinhole have the same size, the focal distance $\mathrm{f}$ considered is $\mathrm{f}=75 \mathrm{~mm}$. In the test and developing process of the camera obscure detector, as a first stage, we have performed several measurements at the 
Sierra Negra Volcano in Puebla-Mexico, at a height of 4200 meters above sea level approximately due to the small light pollution and less atmospheric absorption.

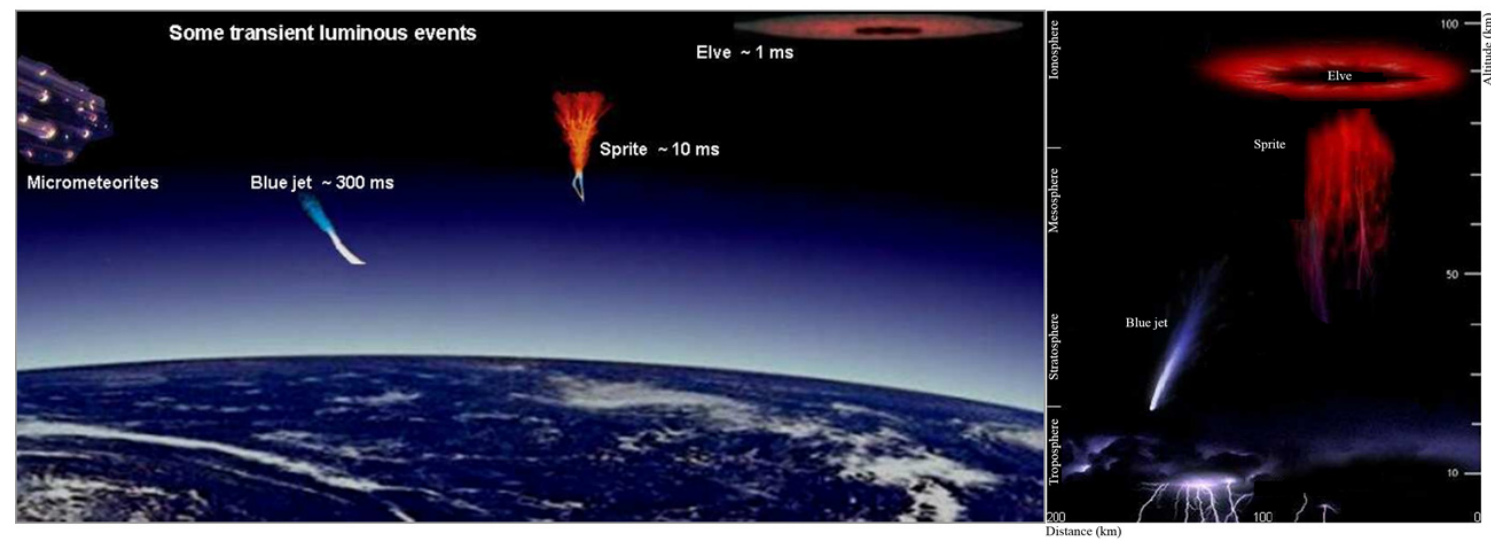

Figure 1: Some transient luminous events measured by video cameras. The images correspond to an elve red sprite, blue jet and micrometorites.
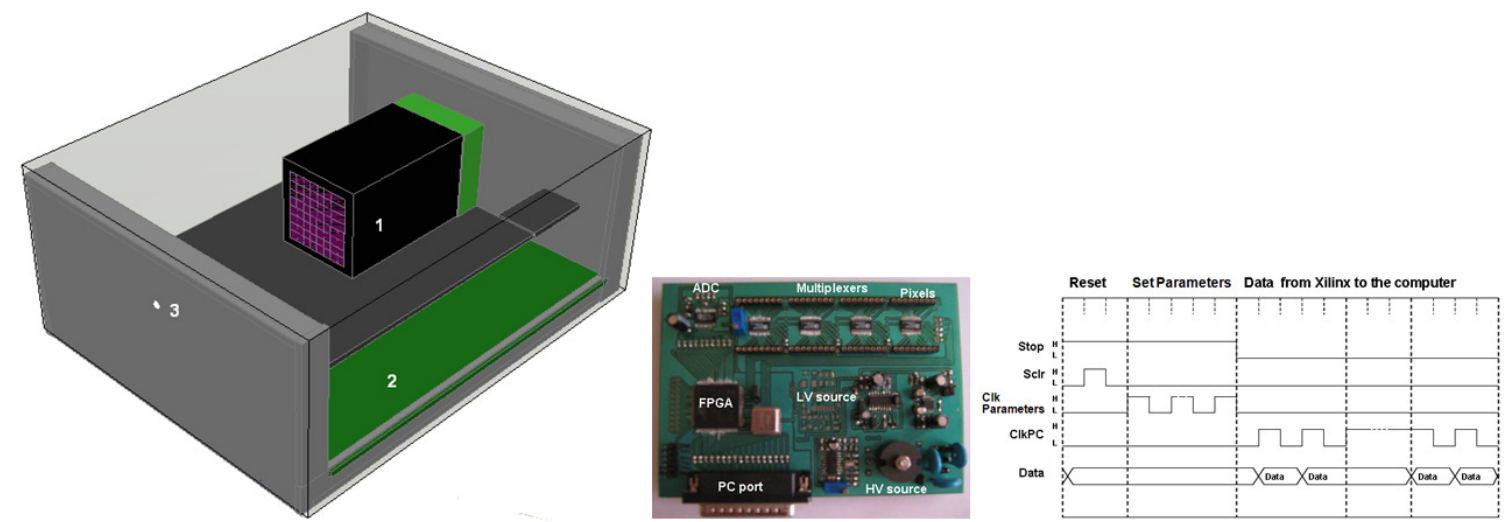

Figure 2: Camera obscura: 1 Photo receiver. 3 pinhole. 2 Electronic Board, all main electrinic parts at center and at right shows the tx-rx data and commands of the camara obscura board and a PC.

\section{Camera electronics}

The figure 3, shows the block diagram and at center of figure 2 the electronic board used to the signal processing of MaPMT when a luminous event will appears. The board has as a main processor a FPGA Xilinx series. This FPGA control the multiplexing and the digitalization of the 64 analog signals from each MaPMT. Also control and monitored the high voltage supplied to the MaPMT in order to protect it if a bright or long lasting event appears. The FPGA stores all the configuration and operation parameters from the $\mathrm{PC}$ and communicate it thru comunication port. The command controls of FPGA are defined by the user by software graphic programmable interface. With the same software we construct a data base in order to process and plot the registered events. The 64 pixels of MAPMT are digitalized with only one ADC. For this we use an array of four multiplexers of type ADG706, where each multiplexer selects 1 signal from its 16 input signals, according to digital values applied for control multiplexing. Then with the array of 4 multiplexer boards we obtain and process every signal from MAPMT as shows in figure 3. 


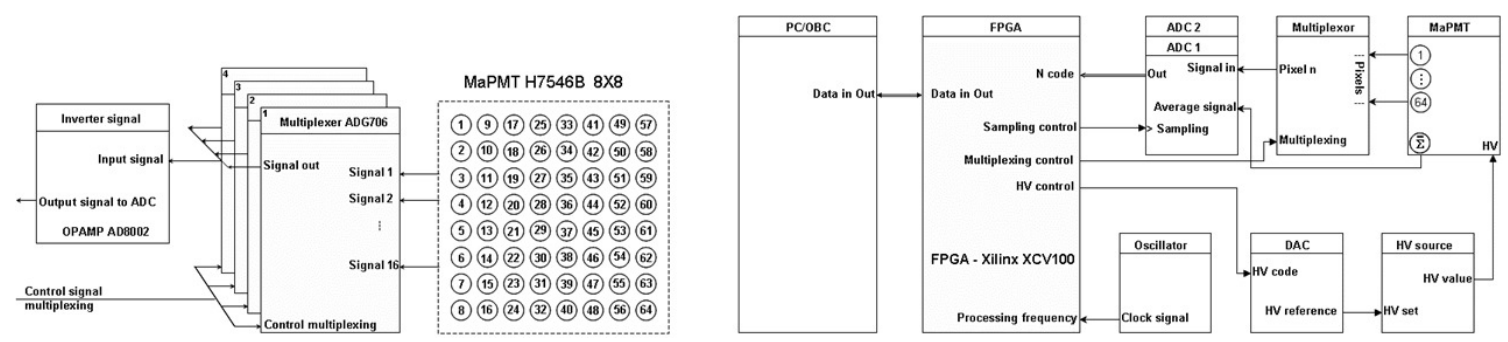

Figure 3: Form of process all the 64 MaPMT's analog signals. At lefth, multiplexing module. at right electronic block diagram.

\section{Camera testing}

As a first step in the calibration process of the Camera Oscura, it is necessary to measure the response to a single photo-electron by the MaPMT [4], and use it to convert the signal to physical photon flux. To obtain the single photoelectron response, we have found the optimal operative voltage for the MaPMT used. To do this, we obtained the single photo electron (SPE) spectrum as a function of supplied voltage with a controlled LED pulse. We selected $950 \mathrm{~V}$ as operative voltage. From the analysis of some thousands events, we found the mean charge produced by a single photo electron response and the SPE pulse amplitude distribution. The designed pinhole camera after calibrated, was tested at mountain Sierra Negra in Puebla-Mexico located geographically at a height of 4200 meters above sea level approximately, some results obtained are shown in the next figures.

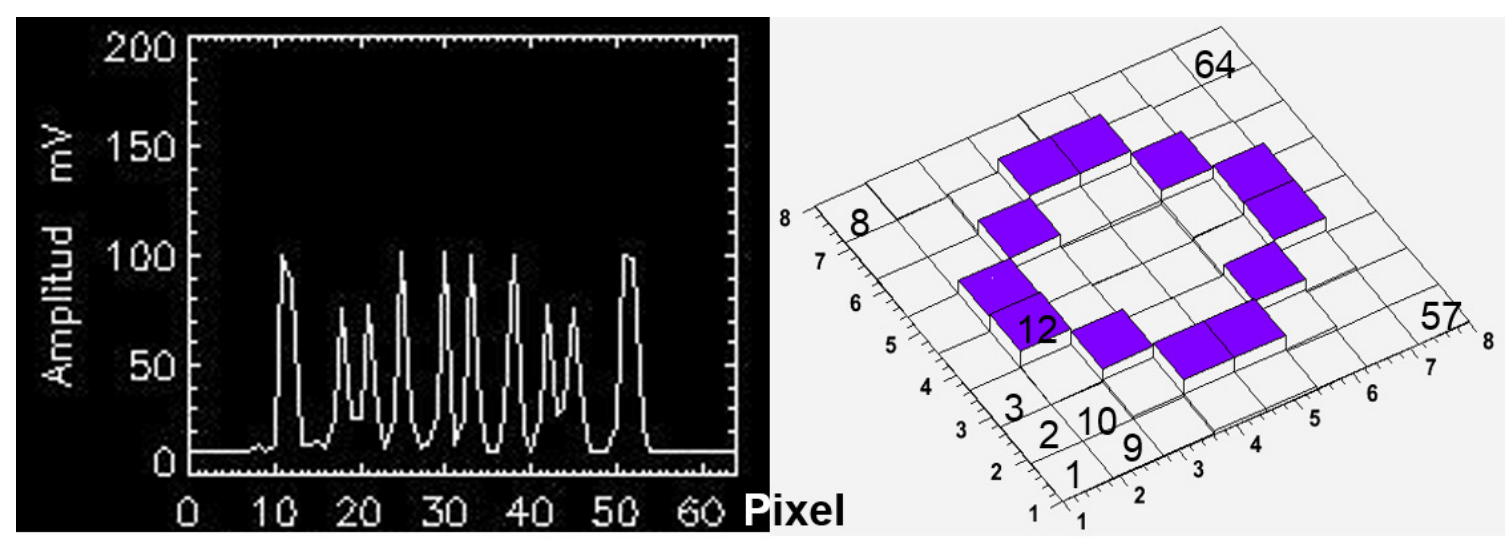

Figure 4: UV TLE registered with pinhole camera in a frame of $64 \mu \mathrm{s}$. Amplitude of each pixel at lefth. At right TLE's image, the numbers corresond to the pixel. 


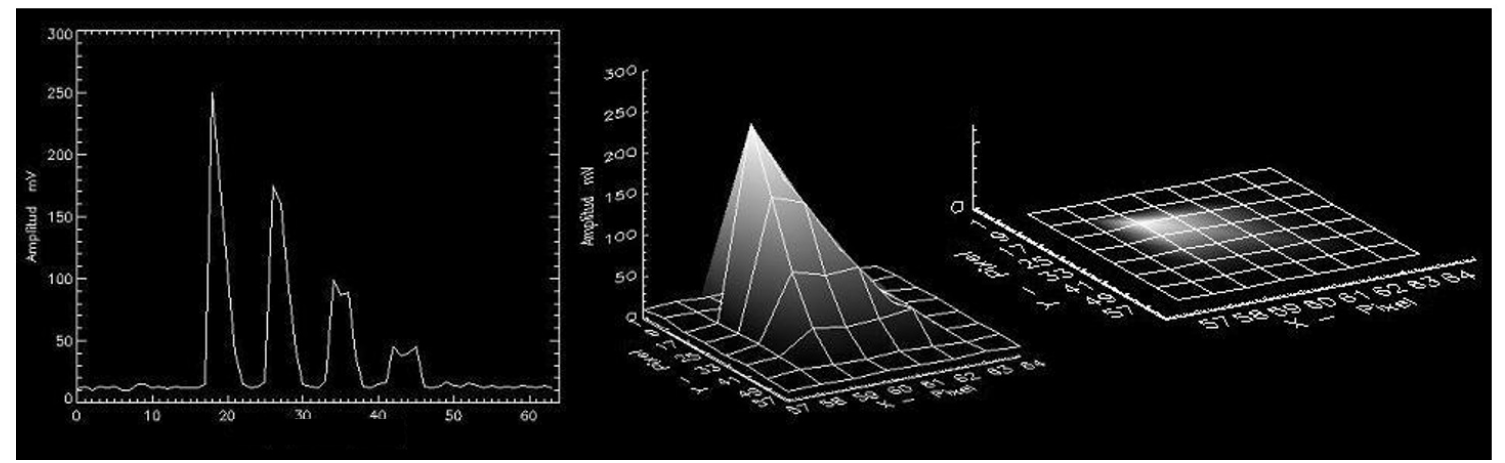

Figure 5: UV TLE registered with pinhole camera in a frame of 32 milliseconds. At lefth, amplitud of each pixel. At center, amplitude distributed in an array of 8x8. TLE's bright, at right.
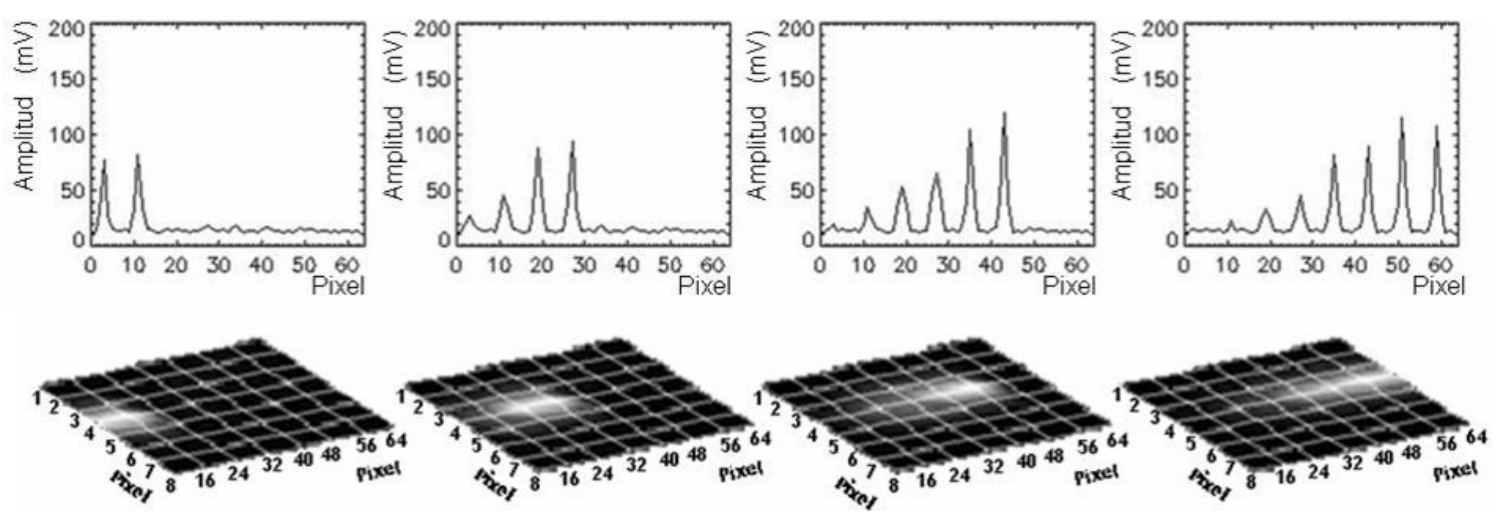

Figure 6: Possible micrometeorite trajectory observed during one second in the pinhole's field of view, in four frames of .25 seconds each one. Amplitude of each pixel, upper. TLE's bright, down. 


\section{Conclusions}

The pinhole camera design has shown to be a fruitful configuration for studies of background light level distribution presented in the atmosphere. The calibration and performance test at the Mexican mountain Pico de Orizaba and Sierra la Negra shows that its possible to detect with high confidence TLE and UV background light.

\section{References}

[1] G K. Garipov, Camera obscura for observation of EAS images in Cerenkov light, J. Phys. G: Nucl. Part. Phys. 20 Printed in the UK [1994] .

[2] G. K. Garipov, Camera obscura network: an array for registration of EAS Cerenkov radiation in the presence of high-intensity sky noise, J. Phys. G: Nucl. Part. Phys. 21 Printed in the UK.

[3] Garipov G.K., Program of transient UV event research at TATIANA-2 satellite, Journal of Geophysical Research A $\mathbf{1 1 5 .}$

[4] Y. Kawasaki., Performance of a multi-anode photomultiplier employing a weak electrostatic focusing system (Hamamatsu R8900 series), Nuclear Instruments and Methods in physics research A $\mathbf{5 6 4}$. 